\title{
CONVERGENCE ACCELERATION BY EXTRACTION OF LINEAR SUBSEQUENCES*
}

\author{
C. BREZINSKI, $\dagger$ J. P. DELAHAYE $\dagger$ AND B. GERMAIN-BONNE $\dagger$
}

Dedicated to Prof. Dr. P. Henrici in honor of his 60 th birthday

\begin{abstract}
Logarithmic sequences are usually difficult to accelerate. In this paper we show how to extract a subsequence converging linearly. This subsequence can be easily accelerated by classical algorithms. Theoretical results are given as well as an algorithmic extraction procedure. Numerical examples illustrate the results.
\end{abstract}

Let $\left(x_{n}\right)$ be a sequence of real numbers converging to $x .\left(x_{n}\right)$ is said to be a linear sequence if $\rho \neq 0, \rho \neq 1$ exists such that

$$
\lim _{n \rightarrow \infty} \frac{x_{n+1}-x}{x_{n}-x}=\rho .
$$

If $\rho=1,\left(x_{n}\right)$ is said to be a logarithmic sequence.

Logarithmic sequences are difficult to accelerate [6], [11], [12], [14]. In this paper we shall present a new idea for accelerating such sequences. It consists in extracting, when possible, a linear subsequence which can be accelerated, for example, by using Aitken's $\Delta^{2}$ process as proved by Henrici [10]. (See also [1], [2], [15].)

Such an idea is surprisingly efficient although it does not make use of all the information contained in the original sequence. Moreover, as we shall see below, such techniques provide a tool for proving some theoretical results that we were not able to prove in another way.

The first problem is to know if the acceleration of a subsequence implies the acceleration of the original sequence. This question will be briefly treated in $\S 1$. In $\S 2$, theoretical results about subsequence extraction are given. In $\S 3$ new accelerable subsets are exhibited. Section 4 deals with algorithmic procedures for extraction. Numerical examples are presented in $\S 5$.

1. Sequence and subsequence acceleration. Let $\left(x_{a(i)}\right)$ be a subsequence of $\left(x_{n}\right)$. Let us consider the sequence transformation $\left(x_{a(i)}\right) \rightarrow\left(T_{i}\right)$, where the computation of $T_{i}$ uses only $x_{a(j)}$ for $j \leqq i$.

We assume that $\left(T_{i}\right)$ converges to $x$ faster than $\left(x_{a(i)}\right)$, that is,

$$
\lim _{i \rightarrow \infty}\left(T_{i}-x\right) /\left(x_{a(i)}-x\right)=0 .
$$

We would like to know if the acceleration of the subsequence will imply the acceleration of the original sequence $\left(x_{n}\right)$. For this purpose let us consider $\left(t_{n}\right)$ defined by

$$
t_{n}= \begin{cases}x_{n} & \text { if } n<a(0) \\ T_{i} & \text { if } a(i) \leqq n<a(i+1) .\end{cases}
$$

$t_{n}$ only depends on $x_{j}$ for $j \leqq n$.

THEOREM 1. Let $\left(x_{n}\right)$ be a monotone sequence of real numbers converging to $x$. Let $a: \mathbb{N} \rightarrow \mathbb{N}$ be strictly increasing and let $\left(T_{i}\right)$ satisfy (1).

\footnotetext{
* Received by the editors July 28,1982 . This research was performed under North Atlantic Treaty Organization research grant 027-81.

† U.E.R. I.E.E.A.-M3, Université de Lille 1, 59655 Villeneuve D'Ascq Cédex, France.
} 
If

$$
\liminf _{i \rightarrow \infty}\left(x_{a(i+1)}-x\right) /\left(x_{a(i)}-x\right)=\alpha>0,
$$

then $\left(t_{n}\right)$ defined by (2) converges faster than $\left(x_{n}\right)$; that is,

$$
\lim _{n \rightarrow \infty}\left(t_{n}-x\right) /\left(x_{n}-x\right)=0 .
$$

Proof. For $a(i) \leqq n<a(i+1)$,

$$
\frac{t_{n}-x}{x_{n}-x}=\frac{t_{a(i)}-x}{x_{a(i)}-x} \frac{x_{a(i)}-x}{x_{a(i+1)}-x} \frac{x_{a(i+1)}-x}{x_{n}-x} .
$$

By the monotonicity of $\left(x_{n}\right)$

$$
\left(x_{a(i+1)}-x\right) /\left(x_{n}-x\right) \leqq 1 .
$$

By (3), for all $i \geqq i_{0}$

$$
\left(x_{a(i)}-x\right) /\left(x_{a(i+1)}-x\right) \leqq 1+\alpha^{-1}
$$

and the theorem follows.

Remark. If $\left(x_{a(i)}\right)$ is either linear or logarithmic, then (3) holds.

2. Theoretical aspects of subsequence extraction. Let us first show that it is always possible to extract a linear sequence from a monotone logarithmic sequence.

THEOREM 2 (existence theorem). Let $\left(x_{n}\right)$ be a monotone logarithmic sequence. For every $\rho \in] 0,1[$ there exists $a: \mathbb{N} \rightarrow \mathbb{N}$ strictly increasing such that

$$
\lim _{i \rightarrow \infty}\left(x_{a(i+1)}-x\right) /\left(x_{a(i)}-x\right)=\rho .
$$

Proof. Let $a(i)=\min \left\{j>a(i-1):\left|x_{j}-x\right|<\rho^{i}\left|x_{0}-x\right|\right\}$ with $a(0)=0$.

Let

$$
\varepsilon_{i}=\frac{\rho^{i}\left(x_{0}-x\right)-\left(x_{a(i)}-x\right)}{x_{a(i)}-x}=\rho^{i} \frac{x_{0}-x}{x_{a(i)}-x}-1>0 .
$$

Since $\left(x_{n}\right)$ is logarithmic and $\rho<1$, then for $i$ sufficiently large

$$
\left|x_{a(i)-1}-x\right| \geqq \rho^{i}\left|x_{0}-x\right| \text {. }
$$

Thus

$$
\varepsilon_{i} \leqq \frac{\left(x_{a(i)-1}-x\right)-\left(x_{a(i)}-x\right)}{x_{a(i)}-x}=\frac{x_{a(i)-1}-x}{x_{a(i)-x}}-1 \underset{i \rightarrow \infty}{\longrightarrow} 0
$$

and

$$
\frac{x_{a(i+1)}-x}{x_{a(i)}-x}=\frac{\rho^{i+1}\left(x_{0}-x\right)}{1+\varepsilon_{i+1}} \frac{1+\varepsilon_{i}}{\rho^{i}\left(x_{0}-x\right)} \underset{i \rightarrow \infty}{\longrightarrow} \rho .
$$

Remark. The assumption on the monotonicity of $\left(x_{n}\right)$ can be removed because it can be easily shown that if $\left(x_{n}\right)$ is a logarithmic sequence, there exists $b: \mathbb{N} \rightarrow \mathbb{N}$ strictly increasing such that $\left(x_{b(i)}\right)$ is monotone and logarithmic.

The result of Theorem 2 is only a theoretical one; it does not provide a practical procedure for extracting such a subsequence because knowledge of $x$ is required. More precisely, an algorithmic method for extracting a linear subsequence cannot 
exist for all monotone logarithmic sequences since, otherwise, it will violate a consequence of the remanence property [5], [6].

Let $\mathscr{S}$ be a family of sequences and let $H=\left(h_{n}\right), h_{n}: \mathbb{R}^{n+1} \rightarrow\{0,1\}$. We say that $H$ defines the algorithmic extraction procedure on $\mathscr{S}:\left(x_{n}\right) \rightarrow\left(x_{a(i)}\right)$, where

$$
\{a(0), a(1), \cdots, a(i), \cdots\}=\left\{n \in \mathbb{N}: h_{n}\left(x_{0}, \cdots, x_{n}\right)=1\right\} .
$$

THEOREM 3. Let LM be the set of monotone logarithmic sequences. There is no algorithmic extraction procedure on $\mathrm{LM}$ such that for all $\left(x_{n}\right) \in \mathrm{LM}$, the extracted sequence $\left(x_{a(i)}\right)$ is linear.

Proof. Let $H$ be an algorithmic extraction procedure on LM such that for all $\left(x_{n}\right) \in \mathrm{LM}$ the extracted sequence $\left(x_{a(i)}\right)$ is linear. Let $\left(T_{i}\right)$ be the sequence obtained by applying Aitken's $\Delta^{2}$ process to $\left(x_{a(i)}\right)$ :

$$
T_{i}= \begin{cases}x_{a(i)} & \text { for } i=0,1, \\ \frac{x_{a(i)} x_{a(i-2)}-x_{a(i-1)}^{2}}{x_{a(i)}-2 x_{a(i-1)}+x_{a(i-2)}} & \text { for } i \geqq 2 .\end{cases}
$$

By a result proved by Henrici [10], $\left(T_{i}\right)$ converges faster than $\left(x_{a(i)}\right)$. Let $\left(t_{n}\right)$ be the sequence defined by (2). By Theorem 1 the sequence transformation $\left(x_{n}\right) \rightarrow\left(t_{n}\right)$ accelerates the convergence. Such a transformation cannot exist since LM is remanent [5], [6].

3. Accelerable subsets of LM. Since Theorem 3 gives a negative result, we shall try to construct algorithmic extraction procedures for some subsets of LM.

Let $g=(g(n))$ be a known given monotone logarithmic sequence converging to zero, for example $g(n)=1 /(n+1)$. Let $\mathscr{S}_{\mathrm{g}}$ be the set of sequences $\left(x_{n}\right)$ such that there is a $\lambda \neq 0$ such that

$$
\lim _{n \rightarrow \infty}\left(x_{n}-x\right) / g(n)=\lambda .
$$

Obviously $\mathscr{S}_{\mathrm{g}} \subset \mathrm{LM}$. Some subsets of $\mathscr{S}_{\mathrm{g}}$ can be accelerated, as shown by GermainBonne [8]. We shall now give a procedure for accelerating $\mathscr{S}_{\mathbf{g}}$.

THEOREM 4. i) There exists an algorithmic extraction procedure on $\mathscr{S}_{\mathbf{g}}$ such that for all $\left(x_{n}\right) \in \mathscr{S}_{\mathrm{g}}$ the extracted sequence $\left(x_{a(i)}\right)$ is linear.

ii) $\mathscr{S}_{\mathrm{g}}$ is accelerable.

Proof. i) Since $(g(n)) \in$ LM then, by Theorem 2, a linear subsequence $(g(a(i)))$ exists and is explicitly known. For example, if $g(n)=1 /(n+1), a(i)=2^{i}$. It can be easily proved that $\left(x_{a(i)}\right)$ is linear. Moreover,

$$
\lim _{i \rightarrow \infty} g(a(i+1)) / g(a(i))=\lim _{i \rightarrow \infty}\left(x_{a(i+1)}-x\right) /\left(x_{a(i)}-x\right) .
$$

ii) As in Theorem 3, $\left(t_{n}\right)$ can be constructed with the help of Aitken's process. The transformation $\left(x_{n}\right) \rightarrow\left(t_{n}\right)$ accelerates $\mathscr{S}_{\mathbf{g}}$.

In order to obtain more precise results, we shall now assume that more information on $\left(x_{n}\right)$ is known.

Let $G=\left\{g_{1}, g_{2}, \cdots\right\}$ be a set of sequences where $\left(g_{1}(n)\right) \in \mathrm{LM}$. We assume that for $j=1,2, \cdots$

$$
\lim _{n \rightarrow \infty} g_{j}(n)=0
$$

and

$$
g_{j+1}(n)=\varepsilon_{j}(n) g_{j}(n) \quad \text { with } \lim _{n \rightarrow \infty} \varepsilon_{j}(n)=0
$$


As in the proof of Theorem 4, for every $\left.\rho_{1} \in\right] 0,1\left[\right.$ a linear subsequence $\left(g_{1}(a(i))\right)$ can be explicitly extracted such that

$$
\lim _{i \rightarrow \infty} g_{1}(a(i+1)) / g_{1}(a(i))=\rho_{1} .
$$

We assume that for $j=1,2, \cdots$ there exists $\left.\mu_{j} \in\right] 0,1[$ such that

$$
\lim _{i \rightarrow \infty} \varepsilon_{j}(a(i+1)) / \varepsilon_{j}(a(i))=\mu_{j}
$$

Thus

$$
\rho_{j+1}=\lim _{i \rightarrow \infty} g_{j+1}(a(i+1)) / g_{j+1}(a(i))=\mu_{j} \rho_{j}
$$

and we have

$$
0<\cdots<\rho_{2}<\rho_{1}<1 .
$$

Let $\mathscr{S}_{G}$ be the set of sequences such that

$$
x_{n}-x=c_{1} g_{1}(n)+c_{2} g_{2}(n)+\cdots, \quad n=0,1, \cdots
$$

with $c_{1} \neq 0$.

Obviously $\mathscr{S}_{G} \subset \mathscr{S}_{\mathbf{g}_{1}}$ and thus Theorem 4 holds for $\mathscr{S}_{G}$.

A general acceleration algorithm, called the $E$-algorithm, has recently been obtained by various authors in different contexts [3], [9], [13] (see also [15]). This algorithm is a generalization of the well-known Richardson extrapolation process. It consists in transforming a sequence $\left(u_{n}\right)$ into the sequence $\left(E_{k}^{(n)}\right)_{n \geqq k}$, where

$$
E_{k}^{(n)}=\frac{\left|\begin{array}{ccc}
u_{n-k} & \cdots & u_{n} \\
d_{1}(n-k) & \cdots & d_{1}(n) \\
\hdashline d_{k}(n-k) & \cdots & d_{k}(n)
\end{array}\right|}{\left|\begin{array}{ccc}
1 & \cdots & 1 \\
d_{1}(n-k) & \cdots & d_{1}(n) \\
\hdashline d_{k}(n-k) & \cdots & d_{k}(n)
\end{array}\right|}
$$

and where $d_{1}, d_{2}, \cdots$ are given sequences.

The quantities $E_{k}^{(n)}$ can be computed for all $n$ and $k$ by a recursive algorithm, the $E$-algorithm, which avoids the computation of the determinants appearing in the definition of $E_{k}^{(n)}$.

In (5) replacing $u_{i}$ by $x_{a(i)}$ and $d_{j}(i)$ by $g_{j}(a(i))$ we get a quantity now denoted by $T_{k}^{(n)}$. As in (2), we define $\left(t_{k}^{(n)}\right)$ from $\left(T_{k}^{(n)}\right)$. We have:

THEOREM 5. For all $\left(x_{n}\right) \in \mathscr{S}_{G}$ and all $k \geqq 1$,

$$
t_{k}^{(n)}-x=O\left(t_{k-1}^{(n)}-x\right) \quad(n \rightarrow \infty) .
$$

Proof. The subsequence $\left(x_{a(i)}\right)$ extracted by the method previously defined satisfies the conditions of [3, Thms. 5, 7], and the result follows from Theorem 1.

Remark. In the general theory of the $E$-algorithm the auxiliary sequences $d_{j}$ can depend on the sequence $\left(u_{n}\right)$ itself. In our case this is not possible because $(a(i))$ must be a priori chosen.

As an application of Theorem 5 let us consider the particular case where

$$
g_{j}(n)=h_{n}^{p_{j}}
$$


where $0<p_{1}<p_{2} \cdots$ and where $\left(h_{n}\right) \in \mathrm{LM}$ and tends to zero. If

$$
\lim _{i \rightarrow \infty} h_{a(i+1)} / h_{a(i)}=\rho \in[0,1]
$$

then

$$
\lim _{i \rightarrow \infty} g_{j}(a(i+1)) / g_{j}(a(i))=\rho^{p_{i}}
$$

Since $0<\cdots<\rho^{p_{2}}<\rho^{p_{1}}<1$, all the previous conditions are satisfied, and, thus Theorem 5 holds.

For such sequences this idea was implicitly used by Chisholm [4] and Genz [7] for accelerating the trapezoidal rule with steps in arithmetical progression by the $\varepsilon$-algorithm.

4. An algorithmic extraction procedure. We shall now describe an algorithmic extraction procedure which is very efficient from the practical point of view. Unfortunately only few theoretical results about this procedure have been obtained.

The procedure is as follows:

Step 0 . Let $\lambda \in] 0,1[$. Set $a(0):=0, i:=0$.

Step 1. Replace $i$ by $i+1$.

Set $a(i):=a(i-1)+1$.

Go to step 3.

Step 2. Replace $a(i)$ by $a(i)+1$.

Go to step 3.

Step 3. If $\left|x_{a(i)+1}-x_{a(i)}\right| \leqq \lambda^{i}\left|x_{1}-x_{0}\right|$, then go to step 1 .

Otherwise go to step 2.

A property of this procedure is the following.

THEOREM 6. For all $\left(x_{n}\right) \in \mathrm{LM}$ such that $\lim _{n \rightarrow \infty}\left(x_{n+2}-x_{n+1}\right) /\left(x_{n+1}-x_{n}\right)=1$, the subsequence $\left(x_{a(i)}\right)$ extracted by the preceding procedure satisfies

$$
\lim _{i \rightarrow \infty}\left(x_{a(i+1)+1}-x_{a(i+1)}\right) /\left(x_{a(i)+1}-x_{a(i)}\right)=\lambda .
$$

Proof. Let $\lambda \in] 0,1[$. By construction

$$
a(i)=\min \left\{j>a(i-1):\left|x_{j+1}-x_{j}\right| \leqq \lambda^{i}\left|x_{1}-x_{0}\right|\right\}
$$

The rest of the proof is as for Theorem 2 .

We have been unable to prove that $(6) \Rightarrow(4)$ in the general case. However this implication is true for all the examples considered in [14]. For $x_{n}=1 /(n+1)^{\alpha}, \alpha>0$ we have $=\lambda^{\alpha /(\alpha+1)}$.

It is possible to define other extraction procedures based on analogous ideas.

5. Numerical examples. The tables below give some numerical examples. The computations were performed on the mini-computer Sinclair ZX-81 working with 9 decimal digits.

In the third column of each table we give the ratio (6) to show how it tends to $\lambda$. Since $t_{n}=T_{i}$ for $a(i) \leqq n<a(i+1)$ we shall compare $T_{i}$ with $x_{a(i+1)-1}$. 
Example 1.

\begin{tabular}{rrrlc}
\multicolumn{5}{c}{$x_{n}=1 /(n+1)^{2}, \quad \lambda=0.3$} \\
\hline$i$ & $a(i)$ & ratio $(6)$ & $x_{a(i+1)-1}$ & $T_{i}$ \\
\hline 0 & 0 & 0.0648 & - & - \\
1 & 2 & 0.251 & - & - \\
2 & 4 & 0.391 & 0.0278 & 0.0338 \\
3 & 6 & 0.363 & 0.0123 & 0.0130 \\
4 & 9 & 0.310 & 0.00510 & -0.00180 \\
5 & 14 & 0.287 & 0.00207 & -0.00192 \\
6 & 22 & 0.316 & 0.000918 & -0.000283 \\
7 & 33 & 0.301 & 0.0004 & 0.000177 \\
8 & 50 & 0.293 & 0.000173 & -0.0000395 \\
9 & 76 & 0.302 & 0.0000769 & -0.00000723 \\
10 & 114 & 0.305 & 0.0000346 & 0.00000509 \\
11 & 170 & 0.299 & 0.0000154 & 0.000000978 \\
12 & 255 & 0.302 & 0.00000689 & -0.000000701 \\
\hline
\end{tabular}

\section{Example 2.}

\begin{tabular}{rrrcr}
\multicolumn{5}{c}{$x_{n}=\sum_{i=0}^{n} 1 /(i+1)^{2}, \quad x=1.6449340668, \quad \lambda=0.3$} \\
\hline$i$ & $a(i)$ & ratio (6) & $x_{a(i+1)-1}$ & \multicolumn{1}{c}{$T_{i}$} \\
\hline 0 & 0 & 0.082 & - & - \\
1 & 5 & 0.290 & - & - \\
2 & 11 & 0.319 & 1.598 & 1.578 \\
3 & 22 & 0.315 & 1.6196 & 1.6336 \\
4 & 39 & 0.299 & 1.6313 & 1.644966 \\
5 & 73 & 0.304 & 1.6375 & 1.644835 \\
6 & 134 & 0.303 & 1.6409 & 1.6445422 \\
7 & 245 & 0.302 & 1.6424 & 1.6449386 \\
\hline
\end{tabular}

Example 3.

\begin{tabular}{|c|c|c|c|c|}
\hline$i$ & $a(i)$ & ratio (6) & $x_{a(i+1)-1}$ & $T_{i}$ \\
\hline 0 & 0 & 0.067 & - & - \\
\hline 1 & 4 & 0.401 & - & - \\
\hline 2 & 8 & 0.286 & 0.0916 & 0.150 \\
\hline 3 & 18 & 0.304 & 0.0494 & -0.224 \\
\hline 4 & 36 & 0.305 & 0.0270 & 0.00655 \\
\hline 5 & 69 & 0.306 & 0.0149 & 0.000244 \\
\hline 6 & 129 & 0.301 & 0.00812 & 0.000118 \\
\hline
\end{tabular}


$\lambda=0.7$

\begin{tabular}{ccccc}
\hline$i$ & $a(i)$ & ratio $(6)$ & $x_{a(i+1)-1}$ & $T_{i}$ \\
\hline 17 & 44 & 0.689 & - & - \\
18 & 54 & 0.710 & - & - \\
19 & 65 & 0.695 & 0.0238 & 0.0109 \\
20 & 79 & 0.706 & 0.0199 & -0.0151 \\
21 & 95 & 0.696 & 0.0166 & 0.00485 \\
22 & 115 & 0.705 & 0.0139 & -0.00639 \\
23 & 138 & 0.701 & 0.0116 & 0.00299 \\
24 & 166 & 0.697 & 0.00970 & -0.00169 \\
25 & 200 & 0.701 & 0.00812 & -0.000973 \\
26 & 240 & 0.700 & 0.00679 & 0.000843 \\
27 & 288 & 0.702 & 0.00569 & -0.000182 \\
\hline
\end{tabular}

\section{REFERENCES}

[1] C. BREZINSKI, Accélération de la convergence en analyse numérique, Lectures Notes in Mathematics 584, Springer-Verlag, Heidelberg, 1977.

[2] —-, Algorithmes d'accélération de la convergence. Etude numérique, Editions Technip, Paris, 1978.

[3] — A general extrapolation algorithm, Numer. Math., 35 (1980), pp. 175-187.

[4] J. S. R. Chisholm, Accelerated convergence of sequences of quadrature approximants, Second Colloquium on Computational Methods in Theoretical Physics, Marseille, 1971.

[5] J. P. Delahaye AND B. Germain-Bonne, Résultats négatifs en accélération de convergence, Numer. Math., 35 (1980), pp. 443-457.

[6] - The set of logarithmically convergent sequences cannot be accelerated, this Journal, 19 (1982), pp. 840-844.

[7] A. GEnZ, Applications of the $\varepsilon$-algorithm to quadrature problems in Padé Approximants and Their Applications, P. R. Graves-Moris, ed., Academic Press, New York, 1973.

[8] B. GERMAIN-BONNE, Estimation de la limite de suites et formalisation de procédés d'accélération de convergence, Thèse, Université de Lille 1, 1978.

[9] T. HÅvIE, Generalized Neville type extrapolation schemes, BIT, 19 (1979), pp. 204-213.

[10] P. HenRICI, Elements of Numerical Analysis, John Wiley, New York, 1964.

[11] C. KoWALEWSKI, Accélération de la convergence pour certaines suites à convergence logarithmique, in Padé Approximation and Its Applications, Amsterdam 1980, M. G. de Bruin and H. Van Rossum, eds., Lecture Notes in Mathematics 888, Springer-Verlag, Heidelberg, 1981.

[12] — Possibilités d'accélération de la convergence logarithmique, Thèse de Troisième Cycle, Université de Lille 1, 1981.

[13] C. SCHNEIDER, Vereinfachte Rekursionen zur Richardson-Extrapolation in Spezialfällen, Numer. Math., 24 (1975), pp. 177-184.

[14] D. A. SMITH AND W. F. FORD, Acceleration of linear and logarithmic convergence, this Journal, 16 (1979), pp. 223-240.

[15] J. WIMP, Sequence Transformations and Their Applications, Academic Press, New York, 1981. 